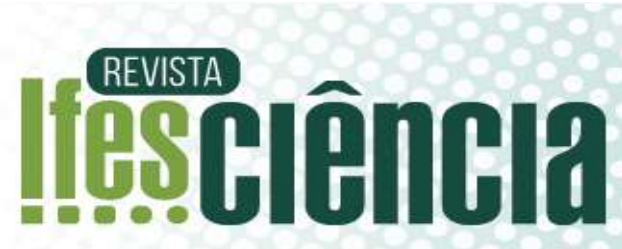

\section{Adequação a programas de controle de qualidade em uma agroindústria familiar do município de Castelo - ES}

\author{
Suitability for quality programs in a family agroindustry in the municipality of \\ Castelo-ES
}

\author{
${ }^{1 *}$ Daiane Bonizioli Benincá \\ ${ }^{2}$ Monique Lopes Ribeiro \\ ${ }^{3}$ Ana Mery de Oliveira \\ ${ }^{4}$ Hudson Cássio Gomes Oliveira
}

\begin{abstract}
${ }^{1}$ Instituto Federal do Espírito Santo. E-mail: daiane-beninca@hotmail.com ${ }^{2}$ Instituto Federal do Espírito Santo. E-mail: monique.ribeiro@ifes.edu.br

${ }^{3}$ Instituto Federal do Espírito Santo. E-mail: ana.mery@ifes.edu.br ${ }^{4}$ Instituto Federal do Espírito Santo. E-mail: hudsoncassio@ifes.edu.br

*Autor de correspondência
\end{abstract}

Artigo submetido em 15/12/2020, aceito em 17/03/2021 e publicado em 15/04/2021.

Resumo: As agroindústrias familiares têm grande impacto social e econômico no Espírito Santo. Os programas de controle de qualidade, como as Boas Práticas de Fabricação (BPF) e os Procedimentos Operacionais Padronizados (POP), são fundamentais para o bom funcionamento das agroindústrias, mas muitas vezes não são aplicados. A partir do exposto, objetivou-se com o presente trabalho avaliar a adequação a RDC n ${ }^{\circ}$ 275, de 21 de outubro de 2002, da Agência Nacional de Vigilância Sanitária, em uma agroindústria familiar de chips (banana, batata doce, mandioca e inhame) no município de Castelo - ES. Como metodologia de estudo, realizou-se uma pesquisa quantitativa descritiva e uma lista de verificação para a coleta de dados foi utilizada. Observou-se que a agroindústria em estudo estava em adequação com a legislação e apresentou conformidade com mais da metade dos requisitos propostos. Entretanto, alguns pontos estavam menos adequados que outros, como é o caso dos manipuladores e da ausência de manuais de BPF ou de POP, que pode ser devido à falta de fiscalização e a falta de pessoal especializado na agroindústria. A partir deste trabalho foi possível concluir que a agroindústria estava adequada às normas de Boas Práticas, mas existiam pontos que precisavam ser melhorados, como a elaboração dos manuais de Boas Práticas de Fabricação e dos Procedimentos Operacionais Padronizados.

Palavras-chave: agroindústria familiar; gestão da qualidade; boas práticas de fabricação.

\begin{abstract}
Family agroindustries have a great social and economic impact on Espirito Santo State. Quality control programs, such as Good Manufacturing Practices (GMP) and Standardized Operating Procedures (POP), are fundamental for the good functioning of agroindustry, but they are often not applied. Based on the above, the objective of this study was to assess the suitability of RDC ${ }^{\circ} 275$, on October 21, 2002, of the National Health Surveillance Agency, in an agroindustry that produces snacks (made of banana, sweet potato, manioc and yams) in the rural area of the municipality of Castelo. As a study methodology, a descriptive quantitative research was carried out and a checklist for data collection was used. It was observed that the agroindustry under study was in compliance with the legislation and regulations, and it was also in compliance with more than half of the proposed requirements. However, some points were less suitable than others, such as the case of the manipulators and the absence of
\end{abstract}


manuals of GMP or POP, which may be due to the lack of inspection and the lack of supervision and the lack of specialized personnel in agroindustry. Thus, it is concluded that the agroindustry was in compliance with Good Practice standards, but there were points that need to be improved, such as the presence of Good Manufacturing Practice manuals and Standardized Operating Procedures.

Keywords: family agroindustry; quality management; good manufacturing practices.

\section{INTRODUÇÃO}

O Estado do Espírito Santo tem sua economia baseada principalmente no agronegócio, representando $30 \%$ do PIB estadual (ESPÍRITO SANTO, 2020). O último censo agrícola realizado pelo Instituto Brasileiro de Geografia e Estatística (IBGE), em 2017, identificou 108.014 estabelecimentos agropecuários, sendo que $74,8 \%$ são de agricultura familiar (BRASIL, 2017), presentes em todo o território capixaba.

Historicamente, o processo de agroindustrialização de produtos agropecuários é caracterizado por grandes unidades industriais. No entanto, a agricultura familiar é considerada uma grande fornecedora de matérias-primas para essas indústrias, vendendo sua produção diretamente ou por meio de "atravessadores", o que compromete sua sustentabilidade econômica. A agroindústria familiar é uma das alternativas econômicas para a permanência dos agricultores e agricultoras familiares no meio rural, em um processo que já faz parte da sua própria história e cultura, destinado ao consumo familiar e/ou a venda de excedentes (PREZOTTO, 2016).

A agroindústria familiar valoriza as tradições e os costumes por meio da comercialização de produtos regionais, cujas receitas tradicionais são repassadas entre gerações, o que vai ao encontro da crescente procura dos consumidores por produtos de origem da agricultura familiar, saudáveis, ecológicos e que valorizem as culturas e tradições locais (PREZOTTO, 2016).

As agroindústrias familiares têm grande impacto social e econômico no Espírito Santo. Muitas delas, além da agroindustrialização de alimentos, também desenvolvem atividades voltadas ao agroturismo, colocando o Estado em posição de destaque no cenário nacional. De acordo com último levantamento do Instituto Capixaba de Pesquisa, Assistência Técnica e Extensão Rural (INCAPER), realizado no ano de 2018, existiam aproximadamente 2024 agroindústrias familiares no estado do Espírito Santo (VINHA e DIAS, 2019).

Com o crescimento do mercado de produtos da agroindústria familiar, a preocupação do consumidor com a segurança dos alimentos, bem como as exigências da legislação sanitária para regularização das agroindústrias, cada vez mais se faz necessário a implantação de programas de controle de qualidade. Esses programas, como as Boas Práticas de Fabricação (BPF), os Procedimentos Operacionais Padronizados (POP) e a Análise de Perigos e Pontos Críticos de Controle (APPCC) contribuem e são indispensáveis para que os produtos oferecidos pelas indústrias e agroindústrias apresentem qualidade elevada e maior controle da cadeia produtiva, aumentando a segurança dos alimentos (DA CRUZ et al., 2006; OLIVEIRA et al., 2016).

Contudo, o conhecimento e a utilização de controle de qualidade dentro das agroindústrias ainda são limitados (VINHA e DIAS, 2019). A partir dessa constatação, entende-se que para uma análise clara da gestão da qualidade em uma agroindústria, o primeiro passo é compreender a visão das pessoas envolvidas nos processos (produtivos e gerenciais) em relação a importância da gestão da qualidade, bem como a análise do nível de aplicação no dia a dia da agroindústria (ORSOLIN, 2006). Além 
disso, quanto a contribuição científica do trabalho, destaca-se que são encontrados poucos trabalhos, sejam nacionais ou internacionais, com foco no controle de qualidade das agroindústrias de alimentos e que estudos deste tipo são necessários para a manutenção da qualidade dos alimentos e da segurança dos consumidores.

Neste contexto, objetivou-se com o presente trabalho avaliar a adequação a RDC n² 275, de 21 de outubro de 2002, da Agência Nacional de Vigilância Sanitária (ANVISA), em uma agroindústria familiar de chips (banana, batata doce, mandioca e inhame) no município de Castelo - ES.

\section{REFERENCIAL TEÓRICO}

\subsection{HISTÓRICO \\ DA \\ AGROINDUSTRIALIZAÇÃO \\ DE ALIMENTOS NO ESPÍRITO SANTO}

O início da agroindustrialização de alimentos no Espírito Santo ocorreu a partir dos excedentes de produção nas propriedades rurais, aliado às habilidades de membros da família na produção de alimentos, e com incentivo de profissionais do Emater (atual Incaper), que trabalhavam conceitos de administração e de aproveitamento dos alimentos nas propriedades (FRADE et al., 2016).

O auge da agroindustrialização de alimentos ocorreu nos anos de 1990, com a organização das mulheres no campo. Com isso, a produção que geralmente era de pães, bolos, doces, compotas e biscoitos, começou a ser vendida de porta em porta, e posteriormente, nas feiras livres. $\mathrm{O}$ aumento da produção desencadeou a necessidade de ampliação das áreas de produção e também o desenvolvimento de sistemas que garantissem a qualidade dos alimentos produzidos e, consequentemente, a segurança dos consumidores. Isso foi facilitado pela implantação do Programa Nacional de Fortalecimento da Agricultura Familiar (PRONAF), em 1996 (FRADE et al., 2016).

\subsection{AGROINDÚSTRIA FAMILIAR}

De acordo com as diretrizes para a formulação da Política Nacional da Agricultura Familiar e Empreendimentos Familiares Rurais, estabelecidas pela Lei 11326 de 2006 (BRASIL, 2006), considerase agricultor familiar e empreendedor familiar rural aquele que pratica atividades no meio rural, atendendo, simultaneamente, aos seguintes requisitos: não detenha área maior do que quatro módulos fiscais; utilize predominantemente mão de obra da própria família nas atividades econômicas do seu estabelecimento ou empreendimento; tenha renda familiar predominantemente originada de atividades econômicas vinculadas ao próprio estabelecimento ou empreendimento; tenha percentual mínimo da renda familiar originada de atividades econômicas do seu estabelecimento ou empreendimento e administre seu estabelecimento ou empreendimento com sua família.

Segundo o Decreto 9.064, de 31 maio de 2017, do Ministério do Desenvolvimento Agrário, empreendimento familiar rural é definido como a "forma associativa ou individual da agricultura familiar instituída por pessoa jurídica" (BRASIL, 2017). Dentro desse mesmo artigo, define-se empresa familiar rural como:

Aquela constituída com a finalidade de beneficiamento, processamento ou comercialização de produtos agropecuários, [...] desde que formada exclusivamente por um ou mais agricultores familiares com inscrição ativa no CAF (Cadastro Nacional da Agricultura Familiar) (BRASIL, 2017).

Além disso, as agroindústrias também podem ser classificadas como agroindústrias individuais ou coletivas. As agroindústrias individuais são geridas por apenas uma família, enquanto as agroindústrias coletivas são formadas por mais de uma família, que se organizam em 
forma de associações, cooperativas ou sociedades empresariais, ou ainda no formato de grupos informais, em que existe uma organização, mas não existe formalização jurídica (VINHA e DIAS, 2019).

A agroindustrialização de alimentos nas zonas rurais representa $91,6 \%$ das agroindústrias encontradas no estado do Espírito Santo (ES) (VINHA e DIAS, 2019), constituindo um importante meio de geração de renda para os agricultores. Além da produção familiar rural, também são produzidos alimentos industrializados que abastecem regiões próximas. Com isso, alguns agricultores passam a atuar em duas etapas da cadeia produtiva: o setor primário (produção da matéria-prima) e o setor secundário (industrialização da matériaprima) (PREZOTTO, 2002), sendo que para $48,3 \%$ das famílias, a atividade agroindustrial é apresentada como sua principal fonte de renda e em 30,7\% como caráter complementar (VINHA e DIAS, 2019).

A matéria-prima utilizada muitas vezes é provinda da propriedade dos agricultores e, em caso de necessidade, comprada de outros produtores da região. É a partir daí que cresce a agregação de valor. Ao invés de vender os produtos primários, que muitas vezes possuem preços baixos e normalmente são mais perecíveis, optam pela industrialização, atribuindo valor ao produto. Além disso, outro ponto que favorece $o$ desenvolvimento das agroindústrias é a proximidade às propriedades rurais, facilitando $\mathrm{o}$ aproveitamento de matérias-primas próximas e a custos menores (PREZOTTO, 2002).

Quanto à mão de obra, normalmente é familiar, tanto na colheita quanto na industrialização. De acordo com Vinha e Dias (2019), as mulheres ainda representam a maior parte de pessoas atuantes nas agroindústrias, mas o número de homens envolvidos no processo vem aumentando. As mulheres geralmente são responsáveis pela etapa de fabricação dos produtos, enquanto os homens respondem pela comercialização, pela obtenção da matériaprima e pela documentação da agroindústria. A utilização de mão de obra familiar é facilitada devido ao volume de produção. Alimentos produzidos em agroindústrias, geralmente, possuem apelo artesanal, com volume de produção baixo e produção não automatizada (PREZOTTO, 2002).

Quanto à renda bruta das agroindústrias individuais do $\mathrm{ES}$, a maioria situa-se em um rendimento maior que 4,5 mil reais mensais. E quanto às categorias de produtos industrializados, agroindústrias do setor de bebidas (suco, polpa, néctares, cachaça, aguardentes, licores, vinhos, fermentados alcoólicos e água de coco), possuem uma média de faturamento maior, seguida de agroindústrias de produtos de origem animal e, por último, pelas agroindústrias de produtos de origem vegetal, exceto bebidas (VINHA e DIAS, 2019).

\subsection{GESTÃO DA QUALIDADE NAS AGROINDÚSTRIAS FAMILIARES}

O controle de qualidade pode ser definido de várias formas. De acordo com Juran (1993), entende-se por qualidade a adequação ao uso. Deming (1982), completa que a qualidade está associada a melhoria contínua em produtos e serviços. Enquanto Crosby (1979) diz que a qualidade está muito relacionada com a conformidade dos produtos e serviços de acordo com as necessidades do cliente. A qualidade também é definida como um conjunto de ações que trazem melhorias ao processo produtivo e é tida como fator indispensável para permanência, tanto da empresa quanto dos produtos, no mercado (CARVALHO, 2005). Já para Champredonde e Muchnik (2012), um conceito subjetivo de qualidade nas agroindústrias, engloba todos os valores simbólicos por trás da produção de alimentos, como a valorização do produto 
artesanal, o conhecimento da origem de produção, o sabor diferenciado e a tradição.

De acordo com Juran (1992), a estruturação de uma gestão da qualidade eficiente necessita de três dimensões: planejamento da qualidade, controle da qualidade e melhoria da qualidade. No planejamento da qualidade inclui-se tudo que é exigido pelos clientes, legislação, consumidores e todos os requisitos demandados para a industrialização do produto. No controle da qualidade, busca-se garantir a qualidade de toda a cadeia de produção, desde a obtenção dos insumos até o produto acabado. Nesta etapa, é imprescindível a visualização dos processos, a existência de procedimentos operacionais padronizados e a identificação e controle dos pontos críticos, além da garantia da conformidade e qualidade do produto final. Já a melhoria da qualidade foca na evolução contínua dos processos produtivos e na identificação e correção de problemas que possam ocorrer durante o processo.

Em um estudo realizado por Soares et al. (2017), no estado do Rio Grande do Sul, verificou-se que existiam diversas razões pelas quais os produtores não aplicavam os conceitos de Boas Práticas de Fabricação. Dentre eles, destacavam-se a falta de perspectiva de continuidade do negócio, em especial devido a saída dos filhos do ambiente rural; os custos elevados; às práticas burocráticas do qual o produtor geralmente não tem conhecimento; e a relação custo-benefício. Já em relação a aplicação de sistemas de gestão de qualidade, Thomas e Fernandes (2020) constataram que as agroindústrias de derivados de cana-de-açúcar da Região Noroeste de Missões - RS não adotavam um cronograma formal de produção, não possuíam um passo a passo (POP) das etapas a serem realizadas no processo de produção e não utilizavam planilhas eletrônicas para controle de custos e de rendimentos. Ainda, segundo os autores, essas medidas, muitas vezes, não eram adotadas devido à falta de conhecimento dos gestores das agroindústrias. Mas, apesar disso, nas agroindústrias estudadas, havia um certo controle das atividades, realizado por anotações em cadernos, e os funcionários da produção possuíam domínio do trabalho que realizavam.

Em outro estudo realizado por Osei Tutu e Anfu (2019), em que foi avaliado a implantação dos sistemas de gestão da qualidade e de segurança de alimentos em cerca de 200 empresas familiares em Gana, $84 \%$ dessas empresas não possuíam nenhum tipo de sistema de gestão da qualidade e de segurança de alimentos implantado. E das que possuíam, a implantação se baseava nas BPF e na ISO 22000. Das empresas avaliadas, $16,5 \%$ possuíam um procedimento de inspeção da chegada dos insumos, $13 \%$ possuíam procedimentos operacionais padronizados e apenas $5,5 \%$ possuíam parâmetros para análise e definição dos pontos críticos do processamento. Osei Tutu e Anfu (2019) e Paiva (2013) ainda destacaram que a maior dificuldade das agroindústrias para a implantação dos sistemas de qualidade e de segurança de alimentos é a falta de treinamento de pessoal, o que influencia diretamente na aplicação dos procedimentos necessários, como os POP, as análises de embalagens e a definição dos pontos críticos de controle.

\section{PROCESSOS METODOLÓGICOS}

A pesquisa do presente estudo foi realizada em uma agroindústria familiar, localizada no município de Castelo - ES. Inicialmente, realizou-se o contato com os proprietários da agroindústria e os objetivos e o cronograma de trabalho foram apresentados e ajustados à realidade concreta da agroindústria.

Realizou-se uma pesquisa quantitativa descritiva. As visitas à agroindústria e a coleta de dados foram concretizadas no período de Abril/2020 a Setembro/2020. Devido à pandemia de 
Covid-19, o número de visitas à agroindústria foi reduzido e foram tomadas medidas de prevenção como o uso de máscara, o distanciamento social e o uso de álcool gel.

\subsection{CARACTERIZAÇÃO AGROINDÚSTRIA FAMILIAR}

DA

A agroindústria foi caracterizada conforme a estrutura organizacional e à adequação quanto aos programas de Boas Práticas de Fabricação (BPF) e os Procedimentos Operacionais Padronizados (POP).

Em relação à estrutura organizacional, foram definidas às atribuições de cada colaborador. Também foi definido o número de funcionários, a área da agroindústria, os produtos fabricados e o mercado (BALDOW, 2013).

\subsection{CARACTERIZAÇÃO DA AGROINDÚSTRIA QUANTO A ADEQUAÇÃO AOS PROGRAMAS DE QUALIDADE}

Quanto à adequação aos programas de BPF e POP, foi utilizada a lista de verificação para estabelecimentos produtores/ industrializadores de alimentos, com o objetivo de avaliar o nível de adequação da agroindústria à $\mathrm{RDC} \mathrm{n}^{\circ} 275$, de 21 de outubro de 2002, da Agência Nacional de Vigilância Sanitária (ANVISA), que dispõe sobre o regulamento técnico de procedimentos operacionais padronizados aplicados aos estabelecimentos produtores/ industrializadores de alimentos (BALDOW, 2013; BRASIL, 2002).

A lista de verificação foi composta de 163 questões divididas em cinco itens: edifícios e instalações (A); equipamentos, móveis e utensílios (B); manipuladores $(\mathrm{C})$; produção e transporte (D); e documentação (E), de acordo com a lista de verificação presente em Brasil (2002). Após a aplicação da lista de verificação, foram calculados os percentuais de atendimento de cada item, bem como o percentual geral, calculado com base na adequação da agroindústria a todos os itens aplicáveis presentes na lista de verificação (Eq. 1).

$\%$ de adequação $=\frac{\sum \mathrm{i}}{n * 100}$

Onde,

$\sum i=$ Soma de todos os itens aplicáveis em adequação;

$n=$ Número de itens da lista.

\section{RESULTADOS E DISCUSSÃO}

A agroindústria em estudo foi classificada como individual, ou seja, gerida por apenas uma família (VINHA e DIAS, 2019), e possuía registro por meio da inscrição do talão do produtor rural. Esta agroindústria produz alimentos de origem vegetal, no formato de chips que atendem ao mercado regional, sendo distribuídos pelo litoral sul do Espírito Santo e nas montanhas capixabas. No portfólio de produtos estão a banana chips, a batata doce chips, a mandioca chips e o inhame chips, todos com sabor salgado.

$\mathrm{Na}$ abertura da agroindústria, os produtores receberam assistência técnica do INCAPER e da Vigilância Sanitária Municipal quanto às legislações que deveriam ser seguidas, ao local onde a agroindústria poderia ser construída e no desenvolvimento da planta. A agroindústria também possui registro na vigilância sanitária, sendo realizada visita anual do órgão. Anualmente, também são oferecidos cursos de BPF a todos os funcionários.

A agroindústria é composta por uma cozinha (área de processamento com área igual a $13,2 \mathrm{~m}^{2}$ ), com duas janelas de vidro teladas e uma sala de embalagem (área igual a $13 \mathrm{~m}^{2}$ ), com três janelas de vidro teladas. No total, a área da agroindústria é de 26,2 $\mathrm{m}^{2}$. 
A área de processamento e a sala de embalagem possuem entradas independentes, mas são interligadas na parte interna. A área de processamento é composta por uma pia para lavagem das mãos, uma pia para lavagem dos vegetais, uma mesa e duas fritadeiras elétricas e na sala de embalagens encontram-se uma seladora elétrica e uma mesa, onde os chips já embalados são armazenados. A área externa possui um banheiro com área total de $2,4 \mathrm{~m}^{2}$ com entrada independente.

O processamento é realizado em estrutura física destinada exclusivamente para a produção dos chips e situa-se dentro do limite da propriedade rural. Desta forma, a agroindústria em estudo enquadra-se dentro dos $83,6 \%$ das agroindústrias do estado do Espírito Santo que realiza a produção em local apropriado e de uso exclusivo (DIAS e VINHA, 2015).

A agroindústria é composta por três colaboradores, sendo que todos atuam na área de produção, inclusive os proprietários. Um dos colaboradores é responsável pela preparação da matéria-prima. Os proprietários (cônjuges) são responsáveis pelo processamento, embalagem, transporte e venda. Além disso, o proprietário também é responsável pela colheita da matériaprima, quando provinda da própria propriedade.

A partir da lista de verificação aplicada, foi possível dimensionar a adequação da agroindústria à legislação vigente. A lista de verificação é uma ferramenta simples e de fácil entendimento que tem como objetivo a coleta de dados de forma estruturada e organizada, em que os dados são autoexplicativos e a visualização é rápida e de fácil interpretação (DOS ANJOS et al., 2012). De acordo com Brasil (2002), foram avaliados os seguintes itens: edifícios e instalações (A); equipamentos, móveis e utensílios (B); manipuladores (C); produção e transporte (D); e documentação (E) (Figura 1).
Figura 1: Porcentagem de adequação da agroindústria de acordo com a lista de verificação de Brasil (2002).

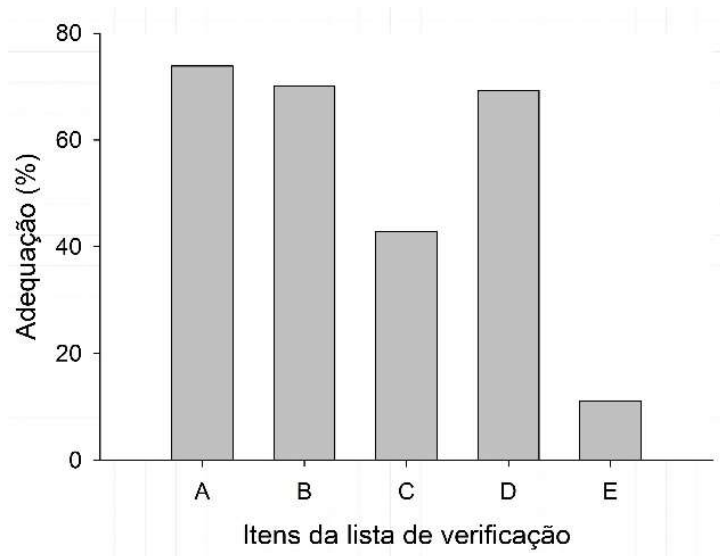

A: edifícios e instalações; B: equipamentos, móveis e utensílios; C: manipuladores; $\mathrm{D}$ : produção e transporte; E: documentação.

Fonte: os autores.

De acordo com as BPF, é imprescindível que o local de fabricação dos alimentos seja adequado e evite contaminações. Os projetos das instalações e edifícios devem permitir o fluxo adequado de pessoas, a separação dos setores (área limpa, área suja e área de embalagem e estocagem), portas e janelas em adequação, entre outros (BRASIL, 2002). Na agroindústria em questão, foram observadas as condições das instalações da agroindústria (A) tanto no ambiente interno (de produção), quanto no ambiente externo (nos arredores), requisitos de higienização, controle de pragas, ventilação, iluminação, áreas de acesso à agroindústria, entre outros. A agroindústria apresentava telas nas janelas; portas, pisos e forros adequados e de fácil higienização, iluminação adequada e lâmpadas com proteção. Entretanto, as portas externas da agroindústria não apresentavam fechamento automático e a água utilizada era coletada de poço artesiano (analisada e aprovada quanto a qualidade pela vigilância sanitária na abertura da agroindústria), visto que no interior do município não existe rede de tratamento de água.

No item B (equipamentos, móveis e utensílios), foram avaliadas as condições higiênico-sanitárias dos objetos, móveis e 
utensílios utilizados no processamento. Os móveis eram dispostos de forma adequada e facilitavam o processamento. Os utensílios que entravam em contato com os alimentos eram de material adequado (inox), higienizados adequadamente $\mathrm{e}$ apresentavam superfície lisa e de fácil higienização, mas não existiam registros de higienização.

No item $\mathrm{D}$ (embalagem $\mathrm{e}$ transporte), foram avaliadas as condições de embalagem, armazenamento e transporte do produto final. Os produtos eram acondicionados adequadamente, em bancadas que apresentavam distanciamento adequado do chão, do teto e das paredes. As embalagens apresentavam-se em conformidade com a legislação, apresentando as informações nutricionais, data de validade, lista de ingredientes, origem do produto, contato e conteúdo líquido (BRASIL, 2020), estando dentro do grupo de $63,1 \%$ das agroindústrias capixabas que comercializam produtos com rótulos, mostrando assim preocupação com a legislação em vigor (DIAS e VINHA, 2015). O transporte era realizado de forma adequada, em carro próprio. Entretanto, não existia programa de amostragem do produto final e a qualidade era definida somente com base no aspecto visual do produto, avaliada pelos próprios produtores.

Pode-se observar que todos esses itens atingiram percentual de atendimento à legislação próximo a $70 \%$, mostrando que existia um controle adequado dessas situações durante o processamento dos chips.

Entretanto, observou-se também que o item C (Manipuladores), que avaliava as condições higiênico-sanitárias dos manipuladores (vestimentas, hábitos higiênicos, uso de equipamento de proteção individual, diálogo excessivo, entre outros) atingiu uma média de $40 \%$ de adequação. Já o item E (Documentação), que abordava sobre a existência de manuais de BPF e POP, atingiu somente $11 \%$ de adequação. $\mathrm{Na}$ agroindústria, os manipuladores apresentavam bom estado de saúde e utilizavam aventais e toucas limpos e conservados. Contudo, não utilizavam sapatos fechados e não haviam cartazes com orientações sobre a lavagem adequada das mãos e manuais de BPF e POP. Em um estudo realizado por Ragazzon (2012), em agroindústrias familiares no Paraná, observou-se também que todas apresentavam deficiências quanto aos treinamentos dos manipuladores em relação a práticas de qualidade e segurança dos alimentos, e quanto a ausência de manuais de BPF.

A baixa adequação no critério dos manipuladores $(\mathrm{C})$ pode estar relacionada tanto à falta dos manuais presentes dentro da agroindústria, quanto à falta de fiscalização/falta de profissionais especializados dentro da agroindústria. Embora haja treinamento oferecido pelo Incaper e Vigilância Sanitária, e fiscalização anual do órgão sobre as BPF, não há um controle frequente de alguns itens. De acordo com Karipidis et al. (2009), a implantação e manutenção dos sistemas de gestão de qualidade nas pequenas indústrias ou agroindústrias ocorrem, em grande parte, devido a exigência da legislação vigente e não em razão do conhecimento dos proprietários sobre os benefícios que esse sistema pode trazer para a empresa. Das empresas que entendem a importância, destacam-se como vantagens o diagnóstico de problemas, a redução de não conformidades, a melhoria dos produtos e serviços, a introdução de novos mercados e as informações sobre qualidade. Como desvantagens evidenciamse a falta de treinamento, os altos custos de certificação, as ferramentas de qualidade insuficientes, o grande volume de documentações, a dificuldade de comprometimento da equipe, a falta de pessoal qualificado e as restrições de tempo e recursos (KARIPIDIS et al., 2009; OSEI TUTU e ANFU, 2019).

Segundo Nascimento Neto et al. (2016), um dos problemas da internalização 
das boas práticas em agroindústrias familiares tem sido a carência de técnicos exercendo a atividade de extensão agroindustrial e a dificuldade de obtenção de informações técnico-operacionais constantes das recomendações de BPF, o que contribui para as não conformidades observadas em auditorias realizadas por organismos da vigilância sanitária.

De acordo com a legislação brasileira, as agroindústrias são classificadas em três grupos, de acordo com a percentagem de adequação a legislação vigente (BRASIL, 2002), nos quais:

- Grupo 1: apresentam 76 a 100\% de adequação aos itens;

- Grupo 2: apresentam 51 a $75 \%$ de adequação aos itens;

- Grupo 3: apresentam de 0 a 50\% de atendimento aos itens.

No parâmetro geral, a agroindústria atendeu a $64 \%$ dos itens previstos na legislação vigente, enquadrando-se no Grupo 2, de acordo com Brasil (2002). Em comparação com agroindústrias avaliadas por Baldow (2013), observou-se que a agroindústria em questão se encontra equiparável às outras. Avaliando a adequação de três agroindústrias do Espírito Santo às normas da ANVISA, verificou-se que uma das agroindústrias estudadas, atuante no setor de laticínios, com treze funcionários, possuía $75 \%$ de adequação, situando-se no grupo 2; outra agroindústria também do setor de laticínio, com nove funcionários, encontrava-se no grupo 1, com aproximadamente $84 \%$ de adequação; e outra agroindústria, que produz alimentos diversificados, de origem animal e vegetal, com quatro funcionários, encontrava-se no grupo 3, com $50 \%$ de conformidade (BALDOW, 2013).

\section{CONCLUSÕES}

A agroindústria avaliada apresentou número de conformidades superior a $50 \%$, sendo superior a estudos anteriores realizados por outro autor em agroindústria de produtos vegetais no ES.

Alguns parâmetros, como edifícios e instalações, equipamentos e utensílios e transporte e embalagens, apresentaram índices de conformidade acima ou igual a $70 \%$.

Entretanto, alguns fatores ainda precisam ser ajustados, como a implantação dos manuais de Boas Práticas de Fabricação e dos Procedimentos Operacionais Padronizados, esse último, principalmente, quanto aos processos de higienização dos equipamentos. Também deve-se destacar a necessidade de mão de obra qualificada para orientação e suporte técnico na agroindústria.

Sugere-se, para trabalhos futuros, a realização de reuniões com os funcionários para ajuste dos itens que apresentaram conformidades inferior a $50 \%$ de adequação, bem como para implantação dos manuais de BPF e POP.

\section{REFERÊNCIAS}

BALDOW, S. G. Planejamento da qualidade em agroindústria de pequena escala. 2013. 104 f. Dissertação de Mestrado, Universidade Federal do Espírito Santo, Alegre, 2013.

BRASIL. Agência Nacional de Vigilância Sanitária. Rotulagem de alimentos. 2020. Disponível em:

https://www.gov.br/anvisa/ptbr/assuntos/alimentos/rotulagem. Acesso em: 06 out. 2020.

BRASIL. Instituto Brasileiro de Geografia e Estatística. Censo Agro 2017:

Resultados definitivos. 2017. Disponível em:

https://censoagro2017.ibge.gov.br/template s/censo_agro/resultadosagro/informativos. html. Acesso em: 13 out. 2020.

BRASIL. Ministério do Desenvolvimento Agrário. Decreto ${ }^{\circ} 9.064$, de 31 de maio de 2017. Dispõe sobre a Unidade Familiar 
de Produção Agrária, institui o Cadastro Nacional da Agricultura Familiar e regulamenta a Lei $n^{\circ} 11.326$, de 24 de julho de 2006, que estabelece as diretrizes para a formulação da Política Nacional da Agricultura Familiar e empreendimentos familiares rurais. Diário Oficial da União: Seção 1. Brasília, DF. 30 mai. 2017. Disponível em: http://www.planalto.gov.br/ccivil_03/_Ato 2015-2018/2017/Decreto/D9064.htm. Acesso em: 15 abr. 2020.

BRASIL. Ministério da Agricultura, Pecuária e Abastecimento. Lei n ${ }^{\circ} 11326$, de 24 de julho de 2006. Estabelece as diretrizes para a formulação da Política Nacional da Agricultura Familiar e Empreendimentos Familiares Rurais.

Diário Oficial da União: seção 1. Brasília, DF, 25 jul. 2006. Disponível em:

http://sistemasweb.agricultura.gov.br/sisleg is/action/detalhaAto.do?method=consultar LegislacaoFederal. Acesso em: 13 out. 2020 .

BRASIL. Agência Nacional de Vigilância Sanitária. Resolução da Diretoria Colegiada - RDC n ${ }^{\circ} 275$, de 21 de outubro de 2002. Dispõe sobre o Regulamento Técnico de Procedimentos Operacionais Padronizados aplicados aos Estabelecimentos Produtores/ Industrializadores de Alimentos e a Lista de Verificação das Boas Práticas de Fabricação em Estabelecimentos Produtores/Industrializadores de Alimentos. Diário Oficial da União: seção 1. Brasília, DF, 23 out. 2002. Disponível em:

http://antigo.anvisa.gov.br/legislacao\#/visu alizar/27002. Acesso em: 20 ago. 2020.

CARVALHO, M. M. Histórico da Gestão da Qualidade. In: CARVALHO, M. M. Gestão da Qualidade: teoria e casos. Rio de Janeiro: Campus, 2005. 376 p.

CHAMPREDONDE, M; MUCHNIK, J. A constructivist view on the quality of food: argentinean experiences. In: ARFINI, F.; MANCINI, M. C.; DONATI, M. (Eds.) Local Agri-food Systems in a Global WorldMarket, Social and

Environmental Challenges. Newcastle: Cambridge Scholars Publishing, 2012. p. $215-243$.

\section{CROSBY, P. Crosby's 14 Steps to}

Improvement. New York: McGraw-Hill. 1979.

DA CRUZ, A. G.; CENCI, S. A.; MAIA, M. C. A. Quality assurance requirements in produce processing. Trends in Food Science and Technology, v. 17, n. 8, p. 406-411, 2006.

DEMING, W. E. Qualidade: a revolução da administração. Rio de Janeiro: Marques Saraiva, 1982. 368 p.

DIAS, R. Q.; VINHA, M. B. Agroindustrialização dos produtos da agricultura familiar do Espírito Santo. Relatório da pesquisa 2013/2014. Vitória: Incaper, 2015. $40 \mathrm{p}$.

DOS ANJOS, M. C.; SOUZA, C. C. DE; CEZAR, I. M.; ARIAS, E. R. A.; REIS NETO, J. F. O uso do método PDCA e de ferramentas da qualidade na gestão da agroindústria no Estado de Mato Grosso do Sul. Revista Agrarian, v. 4, n. 15, p. 7583, 2012.

ESPÍRITO SANTO. Governo do Estado do Espírito Santo. Agronegócio. 2020. Disponível em: https://www.es.gov.br/agronegocio. Acesso em: 13 out. 2020.

FRADE, M. I.; VINHA, M. B.; DIAS, R. $\mathrm{Q}$. Avanços na agroindústria e agroturismo. In: DADALTO, G. G; SILVA, A. E. S. da; COSTA, E. B. da; GAVÊAS, P. A. O.; LOSS, W. R. (Eds.) Transformação da agricultura capixaba: 50 anos. Vitória: Cedagro; Incaper; Seag, 2016. p. $106-114$. 
JURAN, J. M. Planejamento para a Qualidade. 2. ed. São Paulo: Pioneira, 1993. 284 p.

JURAN, J. M. Juran on quality design: the new steps for planning quality into goods and services. New York: Free Press, 1992.548 p.

KARIPIDIS, P.; ATHANASSIADIS, K.; AGGELOPOULOS, S.; GIOMPLIAKIS, E. Factors affecting the adoption of quality assurance systems in small food enterprises. Food Control, v. 20, n. 2, p. 93-98, 2009.

NASCIMENTO NETO, F. do; ALVARENGA, A. L. B.; MACHADO, R. L. P.; DUTRA, A. S. de; MONTEIRO, R. P.; PINTO, M. S. V.; RODRIGUES, A. R. $P$. Manual para internalização das Boas Práticas de Fabricação em agroindústrias familiares. Rio de Janeiro: Embrapa. 2016. 48 p.

OLIVEIRA, C. A. F. de; CRUZ, A. G. da; TAVOLARO, P.; CORASSIN, C. H. Food safety: good manufacturing practices (GMP), sanitation standard operating procedures (SSOP), hazard analysis and critical control point. In: BARROSVELASQUEZ, J. (ed). 1.ed.

Antimicrobial food packaging. Cambridge: Academic Press, 2016. p. 129 $-139$.

ORSOLIN, J. Gestão Da Comercialização $\mathrm{Na}$ Agroindústria Rural Familiar. Revista de Administração, v. 5, n. 8, p. 15-37, 2006.

OSEI TUTU, B.; ANFU, P. O. Evaluation of the food safety and quality management systems of the cottage food manufacturing industry in Ghana. Food Control, v. 101, p. 24-28, 2019.

PAIVA, C. L. Quality Management: Important Aspects for the Food Industry. In: MAZZALUPO, I. (Ed.). Food
Industry. Croácia: InTech Open, 2013. p. $191-218$.

PREZOTTO, L. L. Agroindústria da agricultura familiar: regularização e acesso ao mercado. Brasília: Contag. 2016. $60 \mathrm{p}$.

PREZOTTO, L. L. Uma concepção de agroindústria rural de pequeno porte.

Revista de Ciências Humanas, n. 31, p. 133-153, 2002.

RAGAZZON, D.; SILOCHI, R. M. H. Q.; DE SOUZA LIMA, R. Perfil tecnológico das agroindústrias familiares de Francisco Beltrão - PR. Revista Faz Ciência, v. 14, n. 20, p. 109-124, 2012.

SOARES, R. O.; SIEDENBERG, D. R.; ZAN, F. R.; RUSSO, S. L. Conhecimento tradicionais associados: os entraves e avanços na implantação das Boas Práticas de Fabricação (BPFs) em agroindústrias familiares. In: Congresso International Symposium on Technological Innovation, 8., 2017, Aracaju. Anais[...]. Aracaju: Associação de Propriedade Intelectual, 2017. p. $391-400$.

THOMAS, G.; FERNANDES, D. M. M. A gestão das agroindústrias de melado da Região Noroeste Missões - RS, Brasil, sob a percepção dos seus gestores. Revista Eletrônica Competências Digitais para Agricultura Familiar, v. 6, n.1, p. 123150, 2020.

VINHA, M. B.; DIAS, R. Q. Diagnóstico da Agroindústria Familiar no Espírito Santo: Resultados da Pesquisa 2018. Vitória: Incaper, 2019. 61 p. 